\title{
Comparative analysis of rosaceous genomes and the reconstruction of a putative ancestral genome for the family
}

\author{
Eudald IIla', Daniel J Sargent ${ }^{2}$, Elena Lopez Girona ${ }^{2}$, Jill Bushakra ${ }^{3}$, Alessandro Cestaro ${ }^{4}$, Ross Crowhurst ${ }^{5}$, \\ Massimo Pindo ${ }^{4}$, Antonio Cabrera ${ }^{6}$, Esther van der Knaap ${ }^{6}$, Amy lezzoni ${ }^{7}$, Susan Gardiner ${ }^{3}$, Riccardo Velasco ${ }^{4}$, \\ Pere Arús ${ }^{1}$, David Chagné $^{3}$, Michela Troggio ${ }^{4 *}$
}

\begin{abstract}
Background: Comparative genome mapping studies in Rosaceae have been conducted until now by aligning genetic maps within the same genus, or closely related genera and using a limited number of common markers. The growing body of genomics resources and sequence data for both Prunus and Fragaria permits detailed comparisons between these genera and the recently released Malus $x$ domestica genome sequence.

Results: We generated a comparative analysis using 806 molecular markers that are anchored genetically to the Prunus and/or Fragaria reference maps, and physically to the Malus genome sequence. Markers in common for Malus and Prunus, and Malus and Fragaria, respectively were 784 and 148. The correspondence between marker positions was high and conserved syntenic blocks were identified among the three genera in the Rosaceae. We reconstructed a proposed ancestral genome for the Rosaceae.

Conclusions: A genome containing nine chromosomes is the most likely candidate for the ancestral Rosaceae progenitor. The number of chromosomal translocations observed between the three genera investigated was low. However, the number of inversions identified among Malus and Prunus was much higher than any reported genome comparisons in plants, suggesting that small inversions have played an important role in the evolution of these two genera or of the Rosaceae.
\end{abstract}

\section{Background}

Economically, the Rosaceae is one of the most important plant families [1] comprising some 90 genera with over 3000 distinct species having chromosome numbers ranging from $x=7$ to $x=17$ [2]. Four sub-families are distinguished on the basis of fruit types: the Maloideae (including Malus, and Pyrus); the Prunoideae (Prunus and other stone fruit and almonds); the Rosoideae (Fragaria, Rubus, and Rosa); and the Spiraeoideae (containing many ornamental species, including Physocarpus) [3]. A recent phylogenetic treatment of the Rosaceae based on DNA sequence data of nuclear and chloroplast genomic regions reclassified the genus into three subfamilies (the Dryadoideae, the Rosoideae and the

\footnotetext{
* Correspondence: michela.troggio@iasma.it

${ }^{4}$ IASMA Research and Innovation Centre, Foundation Edmund Mach, Via E

Mach 1, 38010 San Michele all'Adige (TN), Italy

Full list of author information is available at the end of the article
}

Spiraeoideae), each containing a number of distinct supertribes [2]. Prunus and Malus are included in the Spiraeoideae, supertribe Amygdaleae and Pyrodae (tribe Pyrinae) respectively, whilst Fragaria is included in the Rosoideae, supertribe Rosodae (tribe Fragariinae). It has been postulated that the poor phylogenetic resolution along the backbone of the Rosaceae phylogenetic tree suggests a rapid evolutionary radiation of lineages within the family, corresponding to a relatively recent divergence of the genera [2]. Possibly because of the rapid evolution, members of the Rosaceae display remarkable phenotypic diversity, with common morphological synapomorphies not readily identifiable. Indeed, plant habit, chromosome number, and fruit type have all evolved independently on more than one occasion within the family $[2,4,5]$. A better understanding of how the phenotypic diversity within the Rosaceae arose would provide
C Biomed Central

(c) 2011 Illa et al; licensee BioMed Central Ltd. This is an Open Access article distributed under the terms of the Creative Commons Attribution License (http://creativecommons.org/licenses/by/2.0), which permits unrestricted use, distribution, and reproduction in any medium, provided the original work is properly cited. 
an insight into how evolution can lead rapidly to diversification.

Comparative mapping has been carried out in a number of economically important plant families including the Poaceae, Solanaceae, Brassicaceae and Fabaceae [6-10]. In the Poaceae, marker order is highly conserved within syntenic 'genome blocks' between genera [8]. However, despite the conservation of syntenic blocks, grass lineages may rapidly evolve, with high rates of chromosomal 'reshuffling' observed between the rye and wheat genomes [11]. Using restriction fragment length polymorphisms (RFLPs), Bonierbale et al. [12] studied the conservation of synteny between potato (Solanum tuberosum) and tomato (S. lycopersicum syn. Lycopersicon esculentum) and found remarkable conservation of genome structure, with only a few regions where paracentric chromosomal rearrangements could be identified. More recently, Wu and Tanksley [13] reported a higher frequency of inversions than translocations among the genomes of different genera of the Solanaceae.

In the Brassicaceae, almost complete genome colinearity between Arabidopsis thaliana and Capsella rubella has been observed, with gene repertoire, order and orientation highly conserved [14] and likewise, soybean linkage group A2 was shown to be conserved over its entire length with Arabidopsis chromosome I, with just 3 rearrangements identified between the chromosomes of the two species [15]. However, between Arabidopsis and Brassica oleracea, rates of chromosomal rearrangements were shown to be much higher [16]. Between dicotyledenous families, comparisons have been performed between much wider evolutionary distances, for example between Prunus and Arabidopsis [17-19], but only fragmentary patterns of conserved synteny have been observed. However, intrafamilial studies have shown that genome evolution within a family usually proceeds through whole-scale inversions and translocations between chromosomes, meaning regions in which marker order is highly conserved can be identified between genera that diverged millions of years ago, and thus information on genes within conserved genome blocks of one genus can inform studies in other genera within a family.

Comparative genome studies in the Rosaceae have so far been based on the alignment of genetic maps within the same genus, or amongst closely related genera, using small sets of orthologous markers. These studies showed that the genomes of Prunus species are essentially collinear, for example in peach and apricot $[20,21]$, and peach and sweet cherry [22,23]. Similarly, within the Pyrinae tribe, the genomes of Malus, Pyrus and Eriobotrya were shown to be highly collinear [24-26]. Only a few studies investigated genome comparisons across Rosaceae tribes or subfamilies. Dirlewanger et al. [27] compared Malus and Prunus and found strong evidence that single linkage groups in the diploid Prunus were homologous to two distinct homeologous linkage groups in the amphitetraploid genome of Malus. Vilanova et al. [28] compared the diploid reference linkage maps for Prunus (T×E; almond 'Texas' $\times$ peach 'Earlygold') and Fragaria $(\mathrm{FV} \times \mathrm{FN}$; F. vesca '815' $\times$ F. nubicola '601') and they identified numerous chromosomal translocations and rearrangements that occurred in the 29 million years since the genera diverged from a common ancestor. They also found clear cases of conservation of chromosomal synteny, and reconstructed a hypothetical ancestral Rosaceae genome composed of nine chromosomes.

Whole genome sequencing using next generation technologies has now become accessible to the broad scientific community. In the Rosaceae, the genome of Malus $\times$ domestica was recently sequenced using a whole genome shotgun approach [29]. The analysis of the draft sequence of 'Golden Delicious' is consistent with a putative nine chromosome diploid ancestor for the genus [28]. Although there are no published genome sequences that would permit direct comparisons of the M. $\times$ domestica genome and those of other genera of the Rosaceae, there are a large number of genetic markers available for Rosaceous species. Recently, Cabrera et al. [30] reported the development of 857 Rosaceous Conserved Orthologous Set (RosCOS), of which 613 were mapped on the T $\times E$ Prunus reference map. Because of the conserved nucleotide sequence and low or single copy presence across species, the COS sequences are particularly useful for comparative genome studies between related species [30]. The RosCOS set demonstrated extensive conservation of synteny between poplar and Prunus, members of two different plant families in the eurosid clade [30]. Also a significant fraction of the RosCOS markers were transferable to the FV $\times \mathrm{FN}$ Fragaria reference population.

In this paper, we compared the apple genome sequence with conserved molecular markers previously mapped in Prunus and Fragaria [28,30,31], along with an additional set of markers developed for the purpose. The goal was to derive at the ancestral genome structure and organisation of species within the Rosaceae family and to study the genome evolution of the economically relevant Fragaria, Malus and Prunus. The locations of molecular markers on the Prunus and Fragaria genomes were determined through bin mapping [32,33], and their physical positions through analysis of the Malus $\times$ domestica 'Golden Delicious' genome [29]. The comparisons identified syntenic blocks common among the genomes of the three genera as well as within the polyploid Malus genome. The findings allowed us to hypothesize about genome evolution 
within Rosaceae, and to reconstruct its ancestral genome for the family.

\section{Results}

Mapping of markers in the Prunus T×E bin set

A set of transferable expressed sequence tag (EST)derived markers were developed in this study from consensus Prunus-Malus sequences by aligning all available Prunus EST sequences $(75,404$; peach EST database, [34]) to 1,262 Malus 'Golden Delicious' gene sequences with known locations on the $M . \times$ domestica consensus reference map. Of the 155 novel EST-derived markers screened by PCR over the parents of the $\mathrm{T} \times \mathrm{E}$ population, $126(81.2 \%)$ amplified a single band, five (3.2\%) amplified a length polymorphism between the parents of the bin set that was scored by agarose gel electrophoresis, $20(12.9 \%)$ gave no amplification product, and the remaining four (2.4\%) produced multiple fragments. Of the 126 single band markers, those that produced amplicons between 100 and 300 bp (107) were analysed by high resolution melting (HRM). In total, 60 exhibited a clear segregation pattern, enabling their localization on $\mathrm{T} \times \mathrm{E}$ bin map, 17 were monomorphic, and 30 revealed an ambiguous pattern. The 19 PCR marker products larger than $300 \mathrm{bp}$, together with the products of 30 markers that produced ambiguous segregation patterns following HRM analysis, were sequenced directly, enabling a further 29 polymorphic markers to be mapped. Thus in total, 94 of the 155 (60.7\%) new ESTderived markers were located on the Prunus reference map (Table S1 in Additional File 1). Their distribution ranged from one to six markers per bin, and the novel markers were evenly distributed over the eight linkage groups of the T $\times E$ map. Marker MDP0000144421 revealed a new mapping bin $(7: 34)$, however its addition did not increase the coverage of the $\mathrm{T} \times \mathrm{E}$ map.

\section{Mapping of markers in Fragaria $\mathrm{FV} \times \mathrm{FN}$ bin set}

A total of 126 (23\%) RosCOS markers bin mapped in Prunus by Cabrera et al. [30] were located to defined mapping bins on the $\mathrm{FV} \times \mathrm{FN}$ map. Additionally, results from 111 markers previously mapped in Fragaria, Prunus, or in Malus by Vilanova et al. and Sargent et al. $[28,35]$ were included in the analysis. Markers were evenly distributed across the seven $\mathrm{FV} \times \mathrm{FN}$ linkage groups, with 1 - 15 markers located to each bin (Table S2 in Additional File 2).

\section{Marker anchoring to the Malus $\times$ domestica 'Golden Delicious' genome sequence assembly and definition of syntenic blocks}

The sequences of 1,473 markers (572 SSRs, 117 RFLPs, 235 EST-derived markers, and 549 RosCOS), 1321 positioned in Prunus, and 251 in Fragaria, were used as queries for GMAP and BLASTN. Of these, 1,013 markers (214 SSRs, 72 RFLPs, 182 EST-derived markers and 545 RosCOS) showed a high level of DNA sequence conservation with apple, allowing them to be located to precise positions on the Malus chromosomes using GMAP (Table S3 in Additional File 3). In general, a single locus identified in Prunus or Fragaria detected in Malus either one locus, or two distinct homeologues. Following the criteria described in Materials and Methods, 806 markers were retained for syntenic analysis. Of these, 784 were common between Malus and Prunus, whilst 148 were common between Malus and Fragaria and 129 were common to all three genomes (Table 1). Figures 1 and 2 show the relationships between the 806 markers visualized using the Circos program [36]. Figure 1 shows the conservation of synteny between Malus and Prunus, Figure 2 the conservation of synteny between Malus and Fragaria, and Figure S1 in Additional File 4 shows the conservation of synteny between Prunus and Fragaria.

The assignment of collinearity of regions of Malus chromosomes (referred to hereafter as MC) with Prunus and Fragaria was based on the number of reciprocal translocations or fission/fusion events (which we will refer collectively as translocations) and the number of inversions which explain the current marker order in Malus (Table 2). When considering only the common set of 129 syntenic markers, the number of inversions was three times higher between Fragaria and Malus than between Prunus and Malus. At least 45 inversions were needed to place the markers in the same order between Fragaria and Malus, and 14 between Prunus and Malus. When compared with syntenic regions of both Fragaria and Prunus, MC11 and MC7 were characterized by complete collinearity (lack of inversions), based on markers in common across the three genera. When the 784 markers shared only between Malus and Prunus were considered, the number of inversions reached 65 across all $\mathrm{MC}$, almost five times that found with the markers in common across the three genera (Figure S2 in Additional File 5). However, for MC7 and MC11 the numbers of rearrangements observed remained low, with

Table 1 Prunus and Fragaria mapped markers used for synteny comparison with the Malus genome

\begin{tabular}{lccccc}
\hline Marker type & RosCOS & EST & RFLP & SSR & Total $^{\dagger}$ \\
\hline Prunus & 439 & 191 & 49 & 105 & $784(988)$ \\
\hline Fragaria & 90 & 25 & 19 & 14 & $148(164)$ \\
\hline Prunus-Fragaria* & 90 & 20 & 19 & - & 129 \\
\hline Total unique syntenic markers & & & & & 806 \\
\hline
\end{tabular}

*Only markers that were also present in Malus genome sequences were included in the Fragaria-Prunus comparison.

${ }^{\dagger}$ In parentheses, corresponding loci in the Malus whole genome sequence. 


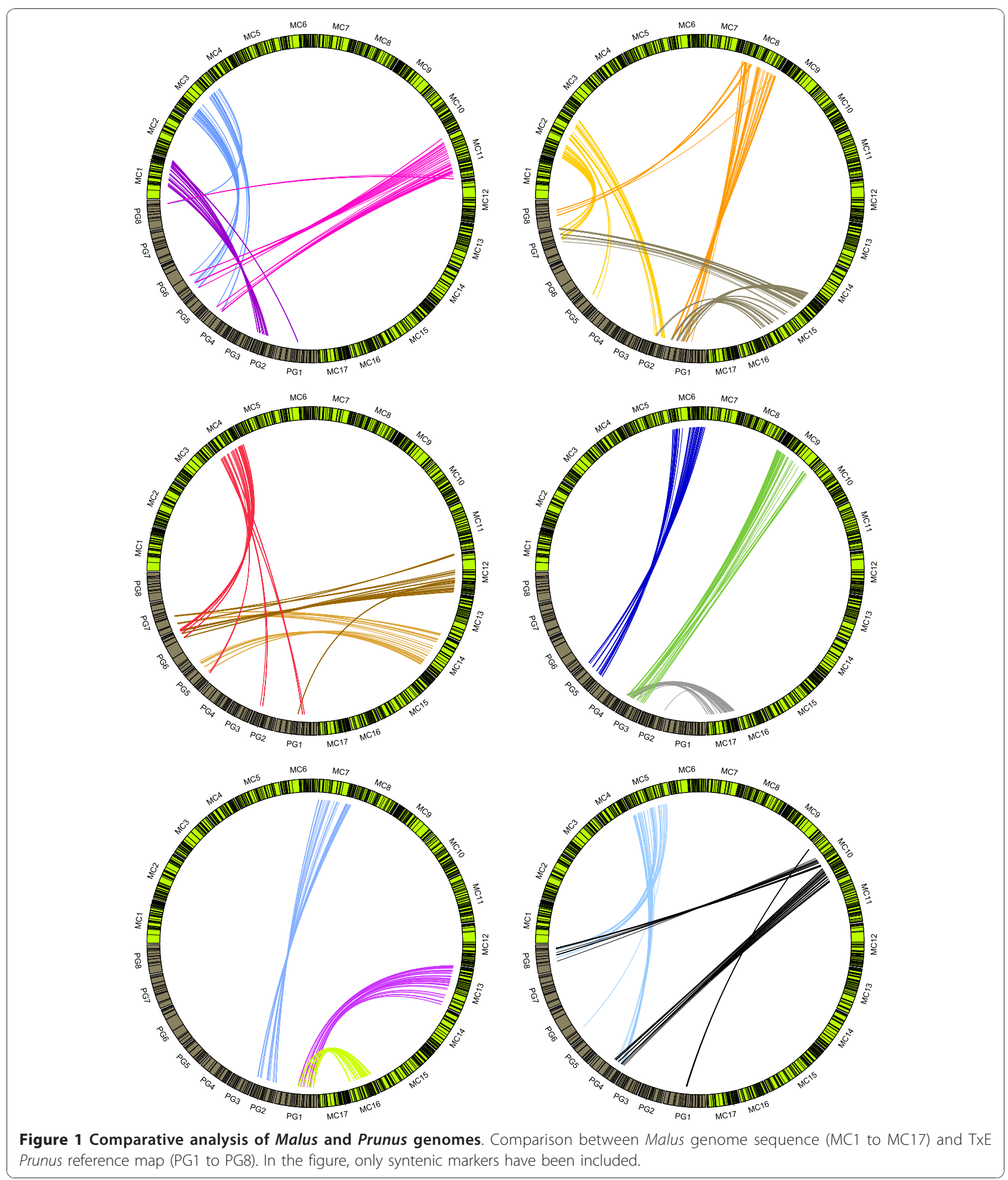

one inversion assigned to each of the two Malus chromosomes. Malus chromosome MC8 was the most rearranged with at least eight inversions required to explain the differences in marker order between Malus and Prunus, and seven between Malus and Fragaria. The total number of translocations between Fragaria and Malus (30) was also higher than that estimated for the PrunusMalus comparison. Certain Malus chromosomes, such as MC2, 4, 12 and 15 were highly rearranged between Malus and the two diploid genomes, with two or three 

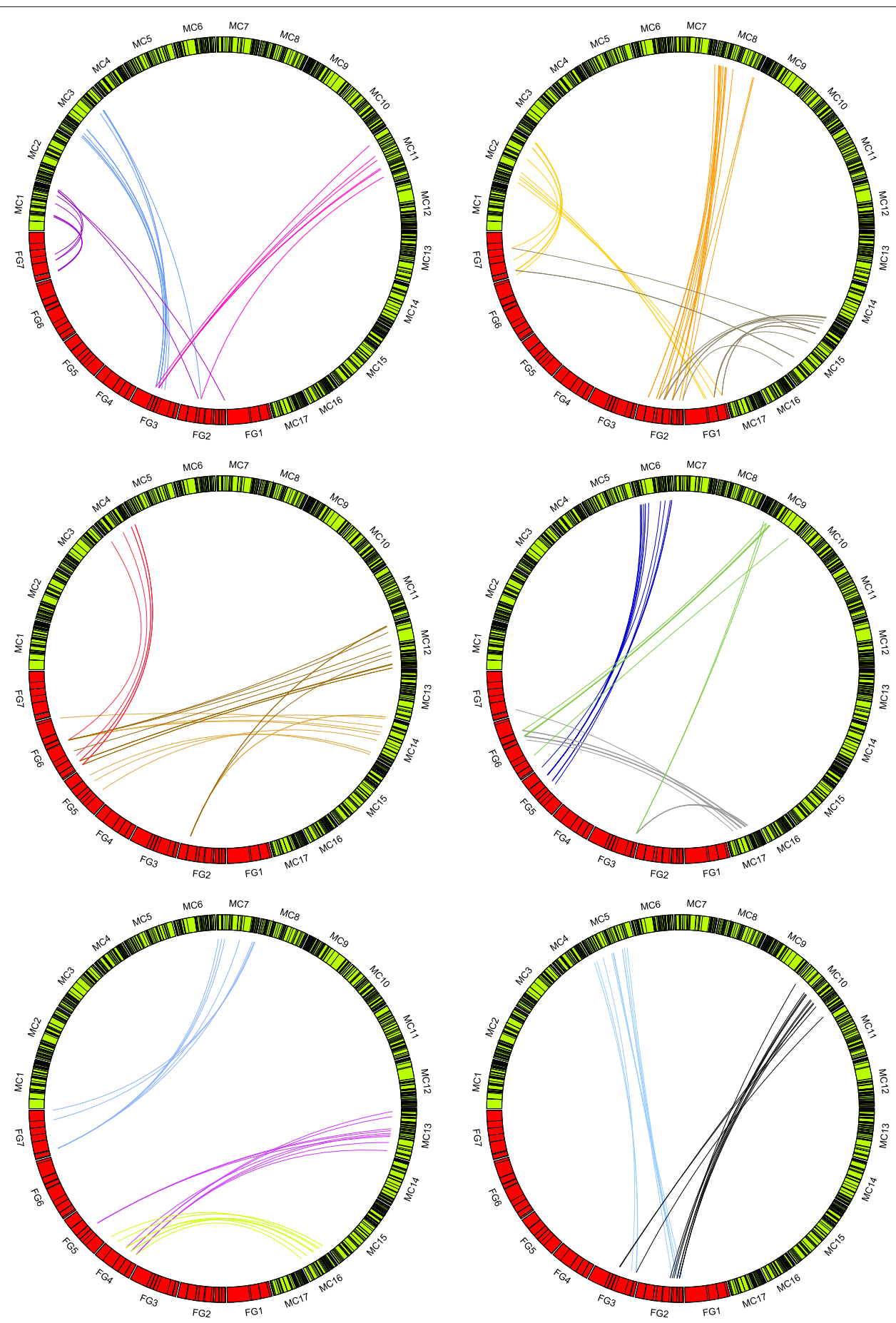

Figure 2 Comparative analysis of Malus and Fragaria genomes. Comparison between Malus genome sequence (MC1 to MC17) and diploid Fragaria FVxFN reference map (FG1 to FG7). In the figure, only syntenic markers have been included.

translocations per chromosome per species, whereas others (MC7 in the comparison with Fragaria and MC6, 7, 9, 13, 16 and 17 in that with Prunus) originated each from a single ancestral DNA fragment. The same translocations were found for Malus-Prunus when using markers shared between the three genera or the complete set of anchor markers.

The locations of markers revealed patterns of conservation of synteny across the genomes of the three genera, as summarized in Figure 3. Within the Malus 
Table 2 Translocations and inversion events hypothesized to occur in Prunus and Fragaria, compared with Malus

\begin{tabular}{|c|c|c|c|c|c|c|}
\hline \multirow[t]{3}{*}{ Malus chromosome } & \multicolumn{4}{|c|}{ Prunus } & \multirow{2}{*}{\multicolumn{2}{|c|}{$\begin{array}{c}\text { Fragaria } \\
\text { Common markers }^{\dagger}\end{array}$}} \\
\hline & \multicolumn{2}{|c|}{ All markers } & \multicolumn{2}{|c|}{ Common markers $^{\dagger}$} & & \\
\hline & Translocations* & Inversions & Translocations* & Inversions & Translocations* & Inversions \\
\hline MC1 & 1 & 6 & 1 & 1 & 1 & 2 \\
\hline MC2 & 2 & 2 & 2 & 0 & 3 & 3 \\
\hline MC3 & 2 & 0 & 2 & 0 & 2 & 4 \\
\hline MC4 & 3 & 3 & 3 & 1 & 3 & 2 \\
\hline MC5 & 1 & 2 & 1 & 0 & 1 & 1 \\
\hline MC6 & 0 & 9 & 0 & 3 & 1 & 4 \\
\hline MC7 & 0 & 1 & 0 & 0 & 0 & 0 \\
\hline MC8 & 1 & 8 & 1 & 4 & 2 & 7 \\
\hline MC9 & 0 & 7 & 0 & 0 & 3 & 2 \\
\hline MC10 & 2 & 3 & 2 & 1 & 2 & 3 \\
\hline MC11 & 2 & 1 & 2 & 0 & 1 & 0 \\
\hline MC12 & 2 & 2 & 2 & 1 & 3 & 3 \\
\hline MC13 & 0 & 4 & 0 & 0 & 1 & 4 \\
\hline MC14 & 1 & 4 & 1 & 1 & 2 & 1 \\
\hline MC15 & 2 & 8 & 2 & 2 & 3 & 5 \\
\hline MC16 & 0 & 4 & 0 & 0 & 1 & 3 \\
\hline MC17 & 0 & 1 & 0 & 0 & 1 & 1 \\
\hline Total & 19 & 65 & 19 & 14 & 30 & 45 \\
\hline
\end{tabular}

*Reciprocal translocations and fission-fusions.

${ }^{\dagger}$ The set of 129 markers in common among the three genomes.

genome, homeologous chromosomes were observed, which corresponded to those identified by Velasco et al. [29] on the basis of whole genome sequence information and reported earlier by Celton et al. [25] on the basis of SSR mapping. Four fully homeologous pairs of chromosomes were revealed: Malus chromosomes 3 and 11; 5 and 10; 9 and 17; and 13 and 16. More complex relationships between chromosomes 4, 6, 12 and 14; chromosomes 1, 2 and 7; and chromosomes 2, 8 and 15 were evident. Among genera, eleven major syntenic blocks were common to all three genomes and, in some cases, conservation of synteny was revealed across whole chromosomes. Amongst these, homeologous groups MC13 and MC16 were syntenic to the top of Prunus linkage group (PG)1 and Fragaria linkage group (FG)4, and the top of Malus homeologues MC5 and MC10 corresponded to PG4 and FG3.

\section{Reconstructing the ancestral genome of the Rosaceae}

A total of 129 markers that mapped to syntenic blocks common to the marker datasets of the three genera were used to develop a model for the ancestral genome of the Rosaceae. The conformation of syntenic blocks shared among the Fragaria, Malus and Prunus genomes was used to model a hypothetical ancestral genome with nine

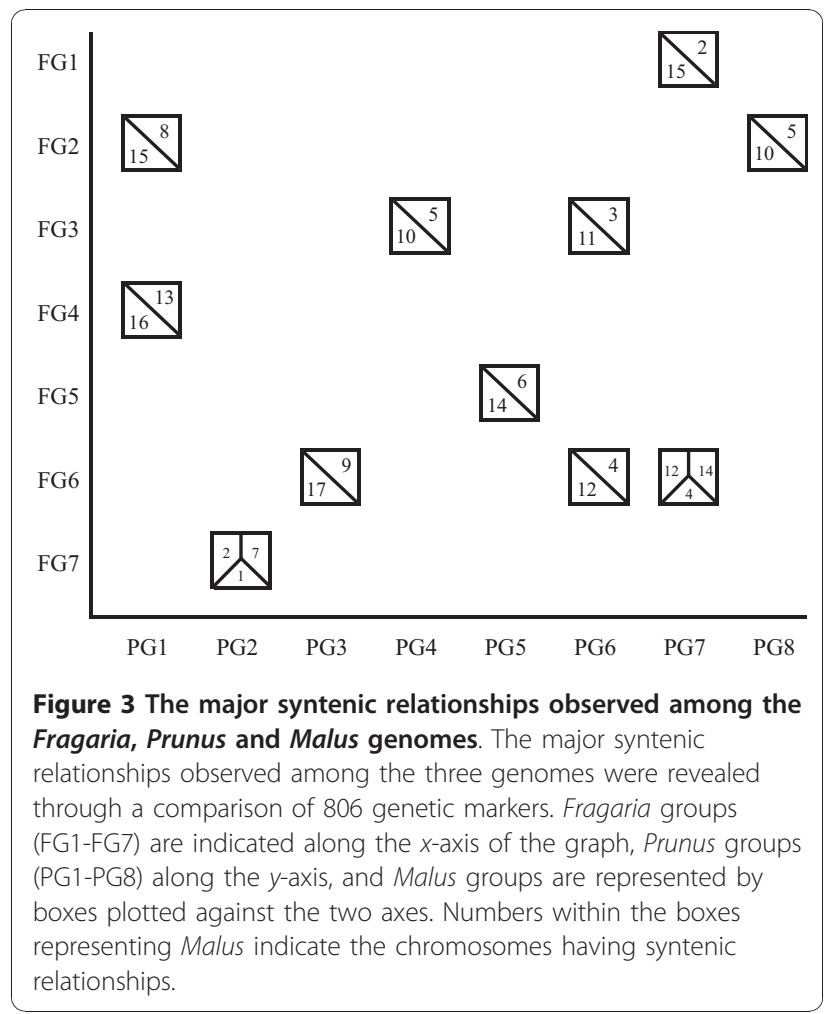




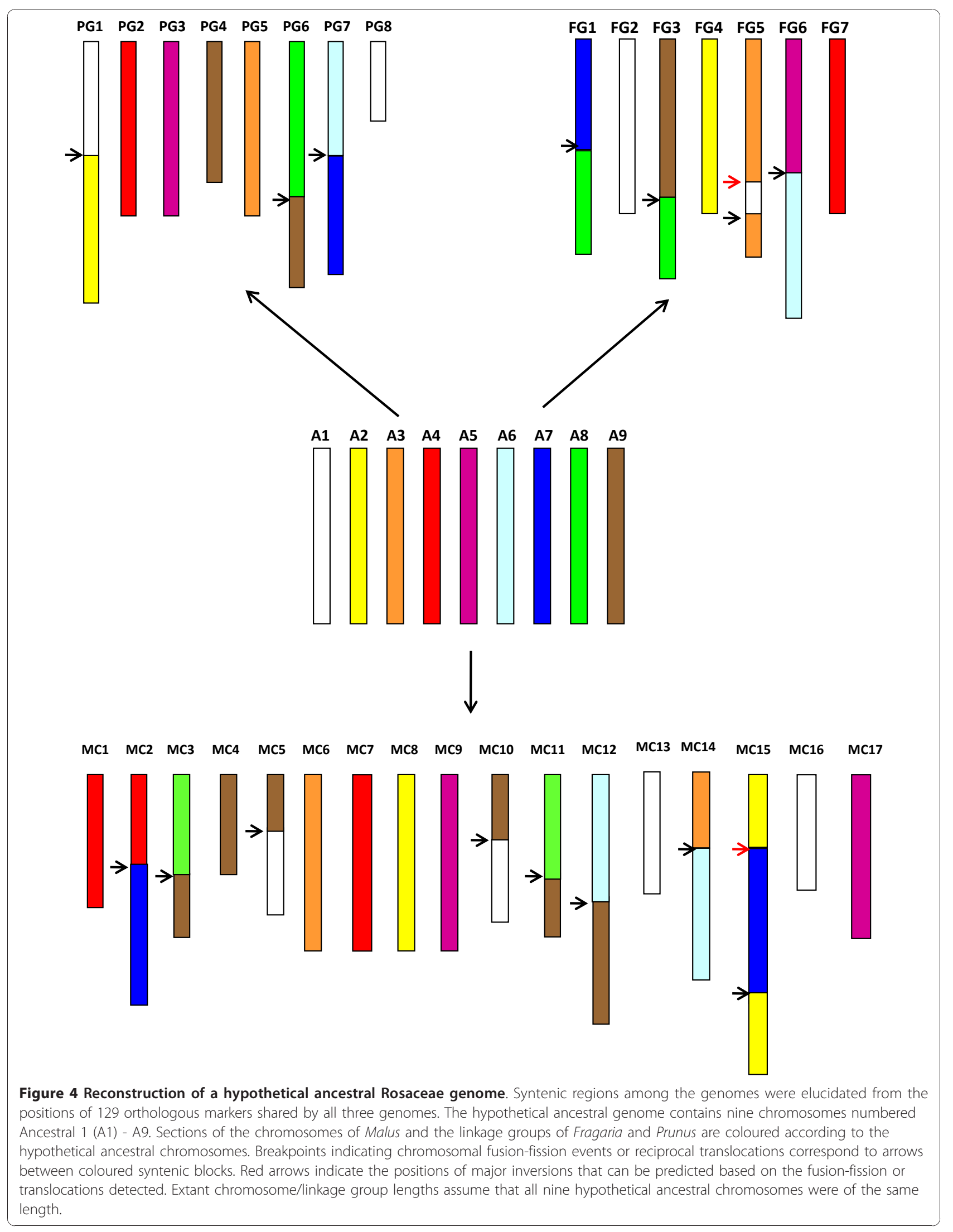


chromosomes (Figure 4). Starting from the ancestral genome and considering only translocation events, the extant Prunus linkage groups $(x=8)$ would have experienced three translocation events, and the Fragaria linkage groups four translocation events, whilst for the Malus chromosomes $(x=17)$, seven translocation events, two of which (a fusion of two major fragments of the two ancestral chromosomes A1 and A9 to form MC5 and MC10 and of A8 and A9 to form MC3 and MC11) preceded the whole genome duplication in apple. Figure 4 depicts the genomes of Fragaria, Malus and Prunus showing the positions of major chromosomal translocations necessary to create their extant genomes starting from the putative nine chromosomes of the ancestral genome. Chromosome/linkage group lengths are approximated relative to the lengths of the hypothetical ancestral chromosomes that we assumed to have a similar length. Colouring of the ancestral chromosomes follows Vilanova et al. [28].

\section{Discussion}

We have conducted a comparative analysis between sequence-characterised molecular markers genetically anchored to the Prunus and Fragaria reference linkage maps and the recently published whole genome sequence of Malus $\times$ domestica [29]. In total, 784 Prunus markers meeting the conservation of synteny criteria described in Material and Methods corresponded to 988 Malus loci. The density distribution of these markers was higher than in previous studies involving other families (one marker every $0.65 \mathrm{cM}$ in Prunus corresponding to one locus every $0.76 \mathrm{Mb}$ in Malus, compared to one marker every $1.6 \mathrm{cM}$ in a comparative analysis between $B$. napus and $A$. thaliana, and one marker every $6.9 \mathrm{cM}$ in a comparison between Capsicum annuum and S. lycopersicum [10,37-42]. The relationships between Fragaria and Malus were based on fewer markers, which revealed 164 loci on the Malus genome sequence (one marker every $4.24 \mathrm{cM}$ in Fragaria corresponding to one locus every 3.64 Mb in Malus). These values, despite being lower than for Prunus, were still within the range of densities employed for genome comparisons based on linkage maps in species of other families.

The RosCOS markers showed the highest level of transferability among genomes, with only 4 out of 549 sequences not found in the Malus genome sequence. This was expected because RosCOS markers have been developed from genes highly conserved across genomes that are present as low or single copy genes in Arabidopsis [30]. Transferability from species to species of EST-derived markers was also high, with $78 \%$ of those tested matching one or two loci in the Malus genome. A similar value was also observed for RFLPs generated from cDNA libraries. In contrast, SSRs derived from genomic regions were less conserved among the three genomes, as reported in previous studies [43]. While SSRs have been shown to be transferable between genera in a family, the evolutionary relationship between target and source genus plays an important role [44]. For example, a higher level of SSR transferability between Prunus and Malus compared with Fragaria and Prunus was expected, since Prunus is phylogenetically closer to Malus than to Fragaria [2]. The results presented in this paper indicate that the majority of genes are conserved among the three genera investigated, however that significantly higher divergence was observed in non-coding regions of the genome than coding. Correspondence among marker/gene positions in the three genomes was high in all pair-wise comparisons, with clear conserved syntenic blocks identified between genera. The 129 markers present in syntenic blocks and common to all three genomes allowed us to propose a hypothetical ancestral genome for the Rosaceae consisting of nine chromosomes. Our study considered nearly twice the number of markers used by Vilanova et al. [28] in an analysis of the FV $\times F N$ and $\mathrm{T} \times \mathrm{E}$ linkage maps. The additional markers revealed an additional translocation event between the hypothetical ancestral chromosomes A1 and A3 to create the extant Fragaria linkage group FG5. The same set of marker data fully support the findings of Velasco et al. [29], who postulated an ancestral diploid progenitor for Malus with a genome containing a chromosome complement of $x=9$. Our data also revealed a fusion/fission event between A1 and A9 during the formation of MC5 and MC10. This occurred before the major whole genome duplication of Malus and thus was not identified in the analysis of the Malus genome alone.

Whilst an early hypothesis as to the origin of Malus implied a wide hybridisation between an ancestral amygdaloid $(x=8)$ and an ancestral spiraeoid $(x=9)$ [45], other data suggest that Malus may have arisen due to a polyploidization of a spiraeoid species $[46,47]$. The more recent hypothesis is supported by molecular phylogenetic analyses based on various nuclear encoded genes [48], and by the comparison between Malus DNA sequence data and that of various Rosaceae species [29], which showed that Gillenia $(x=9)$ was the closest extant diploid genus to both homeologous genomes of Malus. Our results indicate that from the four pairs of homeologous chromosomes entirely conserved in apple, two pairs, MC3-MC11 and MC5-MC10, were assembled from unique major fragments of two ancestral chromosomes. This suggests that the translocations that produced them pre-date the duplication within the apple genome, and were common to the ancestor(s) of apple. The fact that none of these corresponded to rearrangements found in Prunus indicates that its genome is an 
unlikely ancestor of apple. In all, these results support the hypothesis that the two genomes that constitute apple were similar, however distant from Prunus, in agreement with the hypothesis of a possible origin of apple as an autotetraploid from a $x=9$ genome [29]. Hence, evidence from both previous phylogenetic analyses [48] and the comparative mapping data presented here supports the hypothesis that a genome containing nine chromosomes is the most likely most recent ancestral progenitor of the Rosaceae.

Upon pair-wise comparison of each of the three genomes with the ancestral Rosaceae genome (Figure 4), we found that the number of major chromosomal translocations separating them was four, three and eight respectively, which is consistent with a relatively uniform rate of translocation, considering that the apple genome is tetraploid and thus the average number of estimated translocations per genome is four. When using the set of 129 markers common to all three genera, including all rearrangements (major and minor, inversions and translocations) we found that Malus and Prunus differed by 19 translocations and 14 inversions (an average of 9.5 translocations and seven inversions per diploid genome) and Malus and Fragaria by 30 translocations and 45 inversions (15 and 22.5). Nine translocations and 27 inversions were estimated when comparing Fragaria and Prunus [28]. The total number of rearrangements is consistent with genome divergence [2], with less (an average of 16.5) between Malus and Prunus and more in the comparisons involving Fragaria (36-37.5). Our data suggest that the number of inversions (seven for Malus and Prunus and 22 and 27 for the comparisons involving Fragaria) better estimate the evolutionary distance between genera than the number of translocations, as the comparison of inversions between Fragaria and Prunus yields a smaller number (9) than predicted based on the other two comparisons.

When we compared the whole set of 784 markers of Prunus to the Malus genome, the number of translocations identified was the same as when using the 129 markers in common, however the number of inversions was 65 , almost five times higher than that estimated with fewer markers. As expected, most of these inversions were found in relatively small DNA fragments, with $71 \%$ (46) of them concerning apple genomic fragments $\leq 7.5 \mathrm{Mbp}$ ( $1 \%$ of the Malus genome). Other explanations could account for errors in this estimate, such as wrongly-oriented scaffolds in the whole genome apple sequence, or markers misplaced on the linkage maps. It seems however unlikely that they can account for the majority of the inversions observed. When we re-examined our data and discarded 20 markers that, if misplaced would have generated one or more spurious inversions each, we found a more conservative estimate of 43 inversions (more than three times that of the common set of markers), with $58 \%$ of them (25) involving apple DNA fragments $\leq 7.5 \mathrm{Mbp}$, still a very large number of inversions. We attribute this high frequency of inversions to the fact that our comparison is based on an unusually high average density of markers, allowing the identification of much smaller inverted regions compared with other plant families. Paterson et al. [49] did not find such high numbers of inversions in the comparison between the genome sequences of Sorghum bicolor and Oryza sativa and another comparison between a map of $B$. napus with high marker density (0.63 markers/cM) with the genome of Arabidopsis (3.67 markers/Mb) allowed the detection of only eight inversions [39]. A pattern of chromosomal evolution involving many small inversions has been found in Drosophila [50], suggesting that inversions may be an important driving force in the evolution of Rosaceae or at least of the genera investigated, being partly responsible for the high level of phenotypic diversity observed among members of this family.

Based on data from the comparison of genomes of various plant families, Paterson et al. [51] showed that the difference between plant genomes consist of a finite number of chromosomal rearrangements, and estimated average frequency of 0.14 chromosomal rearrangements per Myr of divergence time from a common ancestor. From our data, this may be a reasonable approximation for a low/medium resolution level in terms of anchor points per $\mathrm{Mb}$ of the genome; however, in higher resolution analyses, small inversions may completely change the picture, leading to a variable number of breakpoints depending on the particular history of the genera being compared.

\section{Conclusions}

We have used markers located to the genomes of Fragaria, Malus and Prunus to conduct the first detailed family-wide comparative analysis of the Rosaceae. The high density distribution of markers analysed throughout the genomes of the three genera permitted a detailed study of the conservation of synteny, comparable to those performed in other plant families, such as the Solanaceae. Clear syntenic blocks that were conserved across the family were identified, and a hypothetical ancestral genome for the Rosaceae has been reconstructed using 129 common markers. The hypothetical ancestral genome contained nine chromosomes and was comparable to hypotheses proposed in previous studies of Malus, Fragaria and Prunus [28]. Our study revealed novel syntenic relationships that were not resolved in previous investigations. The number of inversions identified among Malus and Prunus, based on 784 markers was much higher than that 
estimated with fewer markers, and higher than any reported genome comparisons in plants, suggesting that small inversions have played an important role in the evolution of these two genera or of the Rosaceae.

\section{Methods \\ Development and bin mapping of novel EST-derived markers in Prunus}

A total of 155 primer pairs were designed from consensus Prunus-Malus sequences for a set of genes that were well-distributed throughout the 17 Malus linkage groups, using PRIMER 3 [52] with default parameters set to give an expected product size from 150-300 bp with a small number of exceptions. Primers were tested for amplification in the parents of the Prunus reference mapping population ( $\mathrm{T} \times \mathrm{E})$ following Troggio et al. [53].

All primer pairs amplifying a single product of $300 \mathrm{bp}$ or less were used to test for polymorphism between the parental genotypes and the six plants of the $\mathrm{T} \times \mathrm{E}$ bin set [32] using HRM analysis methodology [54] as follows. PCR was performed in a total volume of $10 \mu$ l containing $20 \mathrm{ng}$ of template DNA, $2.5 \mathrm{mM} \mathrm{MgCl} 2,300 \mathrm{nM}$ forward and reverse primers and $1 \times \mathrm{HRM}$ master mix (Roche Applied Science). Both PCR and HRM were performed on a Roche LightCycler ${ }^{\circledR} 480$ (Roche Applied Science). The PCR parameters used were an initial denaturation step of $95^{\circ} \mathrm{C}$ for $10 \mathrm{~min}$, followed by 45 cycles of $95^{\circ} \mathrm{C}$ for $10 \mathrm{~s}, 57^{\circ} \mathrm{C}$ for $15 \mathrm{~s}$, and $72^{\circ} \mathrm{C}$ for $15 \mathrm{~s}$. Following amplification, the samples were heated to $95^{\circ} \mathrm{C}$ for $1 \mathrm{~min}$ and then cooled to $40^{\circ} \mathrm{C}$ for $1 \mathrm{~min}$. Melting curves were generated with continuous fluorescence acquisition during a final ramp from $65^{\circ} \mathrm{C}$ to $95^{\circ} \mathrm{C}$ at $1.1^{\circ} \mathrm{C} / \mathrm{s}$ and the resultant fluorescence data were processed using the LightCycler480 ${ }^{\circledR}$ software (version 1.5.0.39; Roche Applied Science).

Amplicons greater than $300 \mathrm{bp}$ in length and those that gave ambiguous results following HRM analysis were sequenced from the parental genotypes and the bin set following the methods of Troggio et al. [53]. Sequence data were aligned using SEQUENCER v4.8 (Gene Codes Corporation; Ann Arbor, MI, USA), all markers were scored in the bin set and their locations added to the Prunus reference bin map.

\section{Bin mapping of RosCOS markers in Fragaria}

Primer pairs for the 549 RosCOS markers [30] that were successfully anchored to the $M$. $\times$ domestica genome sequence were screened across the parents of the diploid Fragaria mapping population $\mathrm{FV} \times \mathrm{FN}[28,33]$ following the methods of Sargent et al. [55]. Markers that amplified a discrete PCR product were scored in the $\mathrm{FV} \times \mathrm{FN}$ bin set [33] either following agarose gel electrophoresis, or capillary electrophoresis as described in Sargent et al. [55]. Primers which did not reveal visible polymorphisms were extended with a 5' M13F sequence on the forward primer and products were sequenced and scored for single nucleotide polymorphisms (SNPs) as described by Sargent et al. [56].

\section{Marker anchoring to the Malus $\times$ domestica 'Golden Delicious' genome sequence assembly}

The high quality draft genome sequence of $M . \times$ domestica 'Golden Delicious' [29] was used to locate markers bin mapped in Fragaria and Prunus, to the Malus genome. Marker sequences were used as queries for GMAP [57] and as a validation step, BLASTN, using a cut off E-value of $1 \mathrm{e}-15$.

\section{Identification of chromosomal rearrangements and determination of syntenic blocks among Malus, Prunus and Fragaria}

Markers showing a high degree of DNA sequence conservation in apple (BLAST E-value $<\mathrm{e}-15$ ) and that were located to the 'Golden Delicious' genome with GMAP, together with the novel EST-derived mapped markers, were used for the conservation of synteny analysis. Syntenic blocks among Malus, Prunus and Fragaria were defined by the following criteria: a syntenic block should contain a minimum of five (Prunus) or three (Fragaria) homologous marker loci mapping to one or two contiguous bins in Prunus and Fragaria, and located within $3.5 \mathrm{Mbp}$ from each other on the Malus genome. Markers originally mapped in the full populations of either Prunus or Fragaria, were converted to bin positions before the analysis. To visualise the relationships between the genomes of the three genera, the Circos software package was used [36]. As input data for Circos, the physical positions of markers on the Malus genome were divided by $4 \times 10^{5}$ to obtain a genetic distance equivalent to marker linkage values of the Malus consensus map [29]. For the Fragaria and Prunus bin sets, a genetic distance corresponding to the mid-point of each mapping bin was considered. Comparisons between Malus and Prunus, Malus and Fragaria, and Fragaria and Prunus were then visualised, with three linkage groups represented in each ideogram. The method of Vilanova et al. [28] was used to estimate the number of chromosomal rearrangements which occurred among the three genomes since they diverged from a common ancestor.

\section{Reconstruction of a hypothetical ancestral genome for Rosaceae}

The set of 129 genetic markers located on the genomes of all three genera were used to define a hypothetical ancestral genome for the Rosaceae. The positions of these markers were used to identify homeologous chromosomal regions within the Malus genome, and 
subsequently homologous chromosomal segments on the Fragaria and Prunus reference maps. We started with the chromosomal evolution hypothesis of strawberry and peach elaborated by Vilanova et al. [28], modified it with the new data provided by this research, and then constructed the apple chromosome complement using the more complete Prunus-Malus comparison mainly. As we intended to present a simplified view of the complexity of this comparison, we considered translocated segments as only those that involved large DNA fragments (i.e. that covered $\geq 4.41 \mathrm{Mbp}$ of the apple sequence, equivalent to a $10 \%$ of the average apple chromosome $750 \mathrm{Mbp}^{*} 0.10 / 17$ chromosomes). We only included two inversions, one in strawberry and another in apple, that were obvious consequences of the major rearrangements considered in this ancestral genome reconstruction.

\section{Additional material}

Additional file 1: Table S1, novel Prunus EST-derived markers. Table S1 lists locus names, primer sequences, and the TXE bin map positions of the 155 novel EST-derived markers mapped in this work.

Additional file 2: Table S2, Fragaria genetic markers. Table S2 lists the 237 genetic markers of the diploid Fragaria FVXFN reference map, along with their marker type, method of detection and the mapping bins to which they are located.

Additional file 3: Table S3, markers used in this work. Table S3 lists the number and type of markers used in this work. All the available markers were tested against Malus $\times$ domestica genome using GMAP and BLASTN. Syntenic markers are those that fulfilled the criteria defined for conservation of synteny in the Materials and Methods section.

Additional file 4: Figure S1, Prunus and Fragaria map comparison. Figure S1 shows the comparative analysis between Prunus (PG1 to PG8) and diploid Fragaria (FG1 to FG7) reference map using the Circos program. Only markers that were also present in the Malus genome sequences were included in the Fragaria-Prunus comparison.

Additional file 5: Figure S2, Malus and Prunus inversions in the Malus genome. Figure S2 shows the positions in the Malus genome of the inversions detected between Malus and Prunus using the complete set of 784 anchor markers in Prunus. Apple chromosomes are divided in fractions of $10 \mathrm{Mb}$. Arrows indicate the presence of a predicted inversion.

\section{Abbreviations}

RFLP: restriction fragment length polymorphism; SSR: simple sequence repeat; TXE: almond 'Texas' $\times$ peach 'Earlygold'; FVXFN: Fragaria vesca ' $815^{\prime} \times$ Fragaria nubicola '601'; RosCOS: Rosaceous conserved orthologous markers; EST: expressed sequence tag; HRM: high resolution melting; SNP: single nucleotide polymorphisms; MC: Malus chromosome; PG: Prunus linkage group; FG: Fragaria linkage group; WGD: Whole genome duplication.

\section{Acknowledgements}

We gratefully acknowledge Enrico Aldighieri for his assistance in the interpretation of HRM segregation data. We also thank Professor Francesco Salamini for useful comments on a previous version of the manuscript and two anonymous reviewers for their helpful and constructive comments. Apple genome research at FEM is supported by the research office of the Provincia autonoma di Trento. DJS and ELG acknowledge a grant from the East Malling Trust. Fragaria genomics at EMR is funded by the BBSRC. JMB is supported by a grant by Plant \& Food Research's Excellence Programme. Apple genomics at Plant \& Food Research is partially supported by the New
Zealand Foundation for Research Science and Technology project C06X0812 "Exploiting Opportunities from Horticultural Genomics". Research conducted at IRTA was partly funded by the CONSOLIDER-INGENIO 2010 Program (CSD2007-00036) and project INIA-RTA2007-00063-00-00, both from the Spanish Ministry of Science and Innovation. RosCOS development at OSU/ MSU was funded by the National Research Initiative Competitive Grant 200535300-15454 of USDA's National Institute of Food and Agriculture.

\section{Author details}

${ }^{1}$ IRTA, Centre de Recerca en Agrigenòmica CSIC-IRTA-UAB, Carretera de Cabrils Km 2, 08348 Cabrils (Barcelona), Spain. ${ }^{2}$ East Malling Research, New Road, East Malling, Kent ME19 6BJ UK. ${ }^{3}$ The New Zealand Institute for Plant \& Food Research Limited, Palmerston North Research Centre, Private Bag 11600, Palmerston North 4442, New Zealand. ${ }^{4}$ IASMA Research and Innovation Centre, Foundation Edmund Mach, Via E Mach 1, 38010 San Michele all'Adige (TN), Italy. ${ }^{5}$ The New Zealand Institute for Plant \& Food Research Limited, Mt Albert Research Centre, Private Bag 92169, Auckland 1142, New Zealand. ${ }^{6}$ Department of Horticulture and Crop Science, The Ohio State University/Ohio Agricultural Research and Development Center, Wooster OH 44691, USA. ${ }^{7}$ Department of Horticulture, Michigan State University, East Lansing MI 48824, USA.

\section{Authors' contributions}

PA, MT, DJS, DC, SG and RV conceived and designed the experiment. DJS, MT, PA, El, and DC wrote the paper with editorial comment from the other co-authors. El, ELG, JB and MP carried out the experiments and provided interpretation of the results. AC and RC performed the bioinformatics analyses and provided interpretation of the results. ACa, EVK and Al gave access to the RosCOS before publication. All authors read and approved the final manuscript.

Received: 13 August 2010 Accepted: 12 January 2011

Published: 12 January 2011

\section{References}

1. Dirlewanger E, Cosson P, Tavaud M, Aranzana MJ, Poizat C, Zanetto A, Arus P, Laigret F: Development of microsatellite markers in peach Prunus persica (L.) Batsch and their use in genetic diversity analysis in peach and sweet cherry (Prunus avium L.). Theor Appl Genet 2002, 105:127-138.

2. Potter D, Eriksson T, Evans RC, Oh S, Smedmark JEE, Morgan DR, Kerr M, Robertson KR, Arsenault M, Dickinson TA, et al: Phylogeny and classification of Rosaceae. Plant Syst Evol 2007, 266:5-43.

3. Schulze-Menz GK: Rosaceae. In Engler's Syllabus der Pflanzenfamilien. Volume II. Edited by: Melchior H. Berlin: Gebrueder Bornträger; 1964:209-218.

4. Morgan DR, Soltis DE, Robertson KR: Systematic and evolutionary implications of RBCL sequence variation in Rosaceae. Am J Bot 1994, 81:890-903.

5. Potter D, Gao F, Bortiri PE, Oh SH, Baggett S: Phylogenetic relationships in Rosaceae inferred from chloroplast matK and trnL-trnF nucleotide sequence data. Plant Syst Evol 2002, 231:77-89.

6. Kalo P, Seres A, Taylor SA, Jakab J, Kevei Z, Kereszt A, Endre G, Ellis THN, Kiss GB: Comparative mapping between Medicago sativa and Pisum sativum. Mol Genet Genomics 2004, 272:235-246.

7. Lukens L, Zou F, Lydiate D, Parkin I, Osborn T: Comparison of a Brassica oleracea genetic map with the genome of Arabidopsis thaliana. Genetics 2003, 164:359-372.

8. Devos KM, Gale MD: Genome relationships: The grass model in current research. Plant Cell 2000, 12:637-646.

9. Doganlar S, Frary A, Daunay MC, Lester RN, Tanksley SD: A comparative genetic linkage map of eggplant (Solanum melongena) and its implications for genome evolution in the Solanaceae. Genetics 2002, 161:1697-1711.

10. Wu FN, Eannetta NT, Xu YM, Plieske J, Ganal M, Pozzi C, Bakaher N, Tanksley SD: COSII genetic maps of two diploid Nicotiana species provide a detailed picture of synteny with tomato and insights into chromosome evolution in tetraploid N. tabacum. Theor Appl Genet 2010, 120:809-827.

11. Devos KM, Atkinson MD, Chinoy CN, Liu CJ, Gale MD: RFLP-based genetic map of the homoeologous group 3 chromosomes of wheat and rye. Theor Appl Genet 1992, 83:931-939. 
12. Bonierbale MW, Plaisted RL, Tanksley SD: RFLP maps based on a common set of clones reveal modes of chromosomal evolution in potato and tomato. Genetics 1988, 120:1095-1103.

13. Wu FN, Tanksley SD: Chromosomal evolution in the plant family Solanaceae. BMC Genomics 2010, 11:182.

14. Acarkan A, Rossberg M, Koch M, Schmidt R: Comparative genome analysis reveals extensive conservation of genome organisation for Arabidopsis thaliana and Capsella rubella. Plant J 2000, 23:55-62.

15. Grant D, Cregan P, Shoemaker RC: Genome organization in dicots: Genome duplication in Arabidopsis and synteny between soybean and Arabidopsis. Proc Natl Acad Sci USA 2000, 97:4168-4173.

16. Lan TH, DelMonte TA, Reischmann KP, Hyman J, Kowalski SP, McFerson J, Kresovich S, Paterson AH: An EST-enriched comparative map of Brassica oleracea and Arabidopsis thaliana. Genome Res 2000, 10:776-788.

17. Dominguez I, Graziano E, Gebhardt C, Barakat A, Berry S, Arus P, Delseny M, Barnes S: Plant genome archaeology: evidence for conserved ancestral chromosome segments in dicotyledonous plant species. Plant Biotech J 2003, 1:91-99.

18. Georgi LL, Wang Y, Reighard GL, Mao L, Wing RA, Abbott AG: Comparison of peach and Arabidopsis genomic sequences: fragmentary conservation of gene neighborhoods. Genome 2003, 46:268-276

19. Jung S, Main D, Staton M, Cho I, Zhebentyayeva T, Arus P, Abbott A: Synteny conservation between the Prunus genome and both the present and ancestral Arabidopsis genomes. BMC Genomics 2006, 7:81

20. Lambert $P$, Hagen $L S$, Arus $P$, Audergon JM: Genetic linkage maps of two apricot cultivars (Prunus armeniaca L.) compared with the almond Texas x peach Earlygold reference map for Prunus. Theor Appl Genet 2004, 108:1120-1130.

21. Dondini L, Lain O, Geuna F, Banfi R, Gaiotti F, Tartarini S, Bassi D, Testolin R: Development of a new SSR-based linkage map in apricot and analysis of synteny with existing Prunus maps. Tree Genet Genomes 2007, 3:239-249

22. Olmstead JW, Sebolt AM, Cabrera A, Sooriyapathirana SS, Hammar S, Iriarte G, Wang D, Chen CY, van der Knaap E, lezzoni AF: Construction of an intra-specific sweet cherry (Prunus avium L.) genetic linkage map and synteny analysis with the Prunus reference map. Tree Genet Genomes 2008, 4:897-910.

23. Clarke JB, Sargent DJ, Boskovic RI, Belaj A, Tobutt KR: A cherry map from the inter-specific cross Prunus avium 'Napoleon' x P. nipponica based on microsatellite, gene-specific and isoenzyme markers. Tree Genet Genomes 2009, 5:41-51.

24. Yamamoto T, Kirnura T, Saito T, Kotobuki K, Matsuta N, Liebhard R, Gessler C, van de Weg WE, Hayashi T: Genetic linkage maps of Japanese and European pears aligned to the apple consensus map. Proceedings of the XIth Eucarpia Symposium on Fruit Breeding and Genetics 2004, 1 and 2:51-56.

25. Celton JM, Tustin DS, Chagne D, Gardiner SE: Construction of a dense genetic linkage map for apple rootstocks using SSRs developed from Malus ESTs and Pyrus genomic sequences. Tree Genet Genomes 2009, 5:93-107.

26. Gisbert AD, Martinez-Calvo J, Llacer G, Badenes M, Romero C: Development of two loquat Eriobotrya japonica (Thunb.) Lindl. linkage maps based on AFLPs and SSR markers from different Rosaceae species. Mol Breed 2009, 23:523-538.

27. Dirlewanger E, Graziano E, Joobeur T, Garriga-Caldere F, Cosson P, Howad W, Arus P: Comparative mapping and marker-assisted selection in Rosaceae fruit crops. Proc Natl Acad Sci USA 2004, 101(26):9891-9896.

28. Vilanova S, Sargent DJ, Arus P, Monfort A: Synteny conservation between two distantly-related Rosaceae genomes: Prunus (the stone fruits) and Fragaria (the strawberry). BMC Plant Biol 2008, 8:67.

29. Velasco R, Zharkikh A, Affourtit J, Dhingra A, Cestaro A, Kalyanaraman A, Fontana P, Bhatnagar SK, Troggio M, Pruss D, et al: The genome of the domesticated apple (Malus x domestica Borkh.). Nature Genet 2010, 42:833-839.

30. Cabrera A, Kozik A, Howad W, Arus P, lezzoni AF, van der Knaap E: Development and bin mapping of a Rosaceae Conserved Ortholog Set (COS) of markers. BMC Genomics 2009, 10:562.

31. Sargent DJ, Rys A, Nier S, Simpson DW, Tobutt KR: The development and mapping of functional markers in Fragaria and their transferability and potential for mapping in other genera. Theor Appl Genet 2007, 114:373-384
32. Howad W, Yamamoto T, Dirlewanger E, Testolin R, Cosson P, Cipriani G, Monforte AJ, Georgi L, Abbott AG, Arus P: Mapping with a few plants: Using selective mapping for microsatellite saturation of the Prunus reference map. Genetics 2005, 171:1305-1309.

33. Sargent DJ, Cipriani G, Vilanova S, Gil-Ariza D, Arus P, Simpson DW, Tobutt KR, Monfort A: The development of a bin mapping population and the selective mapping of 103 markers in the diploid Fragaria reference map. Genome 2008, 51:120-127.

34. Lazzari B, Caprera A, Vecchietti A, Merelli I, Barale F, Milanesi L, Stella A, Pozzi C: Version VI of the ESTree $\mathrm{db}$ : an improved tool for peach transcriptome analysis. BMC Bioinformatics 2008, 9(Suppl 2):S9.

35. Sargent DJ, Fernandez-Fernandez F, Ruiz-Roja JJ, Sutherland BG, Passey A, Whitehouse AB, Simpson DW: A genetic linkage map of the cultivated strawberry (Fragaria * ananassa) and its comparison to the diploid Fragaria reference map. Mol Breed 2009, 24:293-303.

36. Krzywinski M, Schein J, Birol I, Connors J, Gascoyne R, Horsman D, Jones SJ, Marra MA: Circos: An information aesthetic for comparative genomics. Genome Res 2009, 19:1639-1645.

37. Kaczmarek M, Koczyk G, Ziolkowski PA, Babula-Skowronska D, Sadowski J: Comparative analysis of the Brassica oleracea genetic map and the Arabidopsis thaliana genome. Genome 2009, 52:620-633.

38. Panjabi P, Jagannath A, Bisht NC, Padmaja KL, Sharma S, Gupta V, Pradhan AK, Pental D: Comparative mapping of Brassica juncea and Arabidopsis thaliana using Intron Polymorphism (IP) markers: homoeologous relationships, diversification and evolution of the A, B and C Brassica genomes. BMC Genomics 2008, 9:113.

39. Parkin IAP, Gulden SM, Sharpe AG, Lukens L, Trick M, Osborn TC, Lydiate DJ: Segmental structure of the Brassica napus genome based on comparative analysis with Arabidopsis thaliana. Genetics 2005, 171:765-781.

40. Wu FN, Eannetta NT, Xu YM, Durrett R, Mazourek M, Jahn MM, Tanksley SD: A COSIl genetic map of the pepper genome provides a detailed picture of synteny with tomato and new insights into recent chromosome evolution in the genus Capsicum. Theor Appl Genet 2009, 118:1279-1293.

41. Wu FN, Eannetta NT, Xu YM, Tanksley SD: A detailed synteny map of the eggplant genome based on conserved ortholog set II (COSII) markers. Theor Appl Genet 2009, 118:927-935.

42. Devos KM: Grass genome organization and evolution. Curr Opin Plant Biol 2010, 13:139-145.

43. Rossetto M: Sourcing SSR markers from related plant species. In Plant genotyping: the DNA fingerprinting of plants. Edited by: Henry RJ. CAB International, Wallingford, UK; 2001:211-224.

44. Mnejja M, Garcia-Mas J, Audergon J-M, Arus P: Prunus microsatellite marker transferability across rosaceous crops. Tree Genet Genomes 2010, 6:689-700.

45. Sax K: The origin of the Pomoideae. Proc Am Soc Hort Sci 1933, 30:4.

46. Sterling C: Comparative morphology of the carpel in the Rosaceae. VIII. Spiraeoideae: Holodisceae, Neillieae, Spiraeeae, Ulmarieae. Am J Bot 1966, 53:10

47. Gladkova VN: On the origin of subfamily Maloideae. Botanicheskij Zhurnal 1972, 57:8.

48. Evans RC, Campbell CS: The origin of the apple subfamily (Maloideae; Rosaceae) is clarified by DNA sequence data from duplicated GBSSI genes. Am J Bot 2002, 89:1478-1484.

49. Paterson AH, Bowers JE, Bruggmann R, Dubchak I, Grimwood J, Gundlach H, Haberer G, Hellsten U, Mitros T, Poliakov A, et al: The Sorghum bicolor genome and the diversification of grasses. Nature 2009, 457:551-556.

50. Bhutkar A, Schaeffer SW, Russo SM, Xu M, Smith TE, Gelbart WM: Chromosomal rearrangement inferred from comparisons of 12 Drosophila genomes. Genetics 2008, 179:1657-1680.

51. Paterson AH, Lan TH, Reischmann KP, Chang C, Lin YR, Liu SC, Burow MD, Kowalski SP, Katsar CS, DelMonte TA, et al: Toward a unified genetic map of higher plants, transcending the monocot-dicot divergence. Nat Genet 1996, 14:380-382.

52. Rozen S, Skaletsky H: Primer3 on the WWW for general users and for biologist programmers. Methods Mol Biol 2000, 132:365-386.

53. Troggio M, Malacarne G, Vezzulli S, Faes G, Salmaso M, Velasco R: Comparison of different methods for SNP detection in grapevine. Vitis 2008, 47:21-30. 
54. Chagne D, Gasic K, Crowhurst RN, Han Y, Bassett HC, Bowatte DR, Lawrence TJ, Rikkerink EHA, Gardiner SE, Korban SS: Development of a set of SNP markers present in expressed genes of the apple. Genomics 2008, 92:353-358.

55. Sargent DJ, Hadonou AM, Simpson DW: Development and characterization of polymorphic microsatellite markers from Fragaria viridis, a wild diploid strawberry. Mol Ecol Notes 2003, 3:550-552.

56. Sargent DJ, Marchese A, Simpson DW, Howad W, Fernandez-Fernandez F, Monfort A, Arus P, Evans KM, Tobutt KR: Development of "universal" genespecific markers from Malus spp. cDNA sequences, their mapping and use in synteny studies within Rosaceae. Tree Genet Genomes 2009, 5:133-145

57. Wu TD, Watanabe CK: GMAP: a genomic mapping and alignment program for mRNA and EST sequences. Bioinformatics 2005, 21:1859-1875.

doi:10.1186/1471-2148-11-9

Cite this article as: Illa et al: Comparative analysis of rosaceous genomes and the reconstruction of a putative ancestral genome for the family. BMC Evolutionary Biology 2011 11:9.

\section{Submit your next manuscript to BioMed Central} and take full advantage of:

- Convenient online submission

- Thorough peer review

- No space constraints or color figure charges

- Immediate publication on acceptance

- Inclusion in PubMed, CAS, Scopus and Google Scholar

- Research which is freely available for redistribution

Submit your manuscript at www.biomedcentral.com/submit
Ciomed Central 